\title{
TEM Characterization of Iron-Doped Ceria-Zirconia and Ceria
}

\author{
R. K. Hailstone, ${ }^{*}$ A. G. DiFrancesco, ${ }^{*}$ L.Herder, ${ }^{* *}$ T. D. Allston, ${ }^{* *}$ and K. J. Reed**** \\ * Center for Imaging Science, Rochester Institute of Technology, Rochester, NY 14623 \\ ** Department of Chemistry, Rochester Institute of Technology, Rochester, NY 14623 \\ *** Cerion Energy Corp, One Blossom Rd., Rochester, NY 14610
}

Nanocrystalline $\mathrm{CeO}_{2}$ (nanoceria) has many real and potential applications, such as in catalysis and fuel cells. Its reactivity is connected to its redox chemistry involving the facile $\mathrm{Ce}^{4+} / \mathrm{Ce}^{3+}$ electrochemistry. This allows nanoceria to store oxygen under oxidizing conditions $\left(\mathrm{Ce}^{4+}\right)$ and to release oxygen under reducing conditions $(\mathrm{O}$ vacancies are created, leading to the formation of $\left.\mathrm{Ce}^{3+}\right)$. A quantitative measure of a material's ability to release and take up oxygen is characterized by its oxygen storage capacity (OSC). Measured OSC values of pure ceria are generally less than $25 \%$ of the theoretical value, depending on preparation method and particle size. In order to improve the oxygen storage capacity of $\mathrm{CeO}_{2}$, doping techniques have been used. Of particular concern in the present work is the effect of iron doping.

Aqueous precipitation in the presence of an organic stabilizer was used to prepare suspension-stable nanoparticles of $\sim 3 \mathrm{~nm}$ diameter. Starting materials were the nitrates of the desired metal ions, 28$30 \% \mathrm{NH}_{4} \mathrm{OH}$ and $50 \% \mathrm{H}_{2} \mathrm{O}_{2}$. Figure 1 shows a bright-field TEM image of our nominally 40 mole $\%$ Fe-doped ceria particles along with a SAED pattern typical for our process parameters. The pattern is consistent with the fluorite lattice of $\mathrm{CeO}_{2}$. Fig 2 is a HRTEM image in which the lattice plane spacing is consistent with the (111) and (220) planes of $\mathrm{CeO}_{2}$. Although analytical data shows $\mathrm{Fe}$ is present in our nanoparticles, the electron diffraction shows that there is no detectable crystalline Fe phase. Figure 3 shows a trend in which the lattice parameter decreases as the Fe concentration increases. These data are for $\mathrm{Ce} / \mathrm{Zr} / \mathrm{Fe}$ precipitations and show that at least some of the $\mathrm{Fe}$ has been incorporated into the ceria, probably in $\mathrm{Ce}^{4+}$ sites, since interstitial Fe would likely increase the lattice constant.

On the basis of previous work where 15 to 20 mole $\%$ was the maximum Fe incorporated, we expect some of the iron in our $40 \mathrm{~mole} \%$ nanoparticles will be in a separate phase and SAED results indicate that it is amorphous. So just what is the amorphous iron oxide phase? We can get some hints to what it might be by considering some precipitation formulations that give a turbid suspension, rather than the clear suspensions leading to the above results. These samples are characterized by large particles, in addition to the small nanoparticles normally produced. These larger particles are often crystalline and an example is given in Fig. 4. The SAED pattern is consistent with a type of $\mathrm{FeOOH}$ phase known as 6-line ferrihydrite $(6 \mathrm{~L} \mathrm{FeHyd).} \mathrm{These} \mathrm{results} \mathrm{give} \mathrm{us} \mathrm{a} \mathrm{clue} \mathrm{that} \mathrm{the}$ amorphous phase is likely an amorphous FeHyd and this would be consistent with other work using precipitation parameters similar to ours which also postulated that amorphous FeHyd was formed during attempts to dope ceria at high Fe levels.

Ferrihydrite can be converted into on iron oxide phase by dehydration and this process can be accelerated by heating. Using a heating sample holder in our TEM, we were able to achieve this conversion. Figure 5 shows an image of the sample before and after heating for $8 \mathrm{~h} \mathrm{at} 470{ }^{\circ} \mathrm{C}$. After heating it is evident that the particles have undergone sintering. The SAED pattern of the heated sample is shown in Fig. 6 and is consistent with ceria, as existed before the heating, and heatinduced crystalline phases consisting of maghemite, which is suggested to be more stable than hematite at small particle sizes, and 6L FeHyd. Such a mixture is consistent with previous work on heated ferrihydrite material in the temperature range used in our work and is further evidence that we have an amorphous ferrihydrite phase in our as-precipitated materials. 

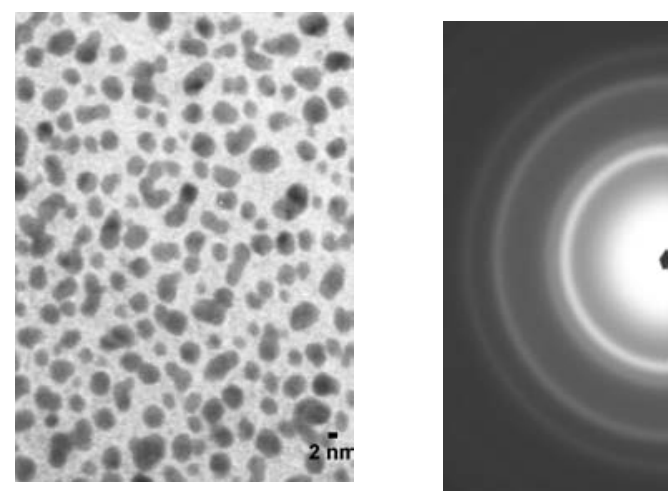

Fig. 1. Low resolution bright-field image of Fe-doped ceria, along with an SAED pattern consistent with the fluorite structure of ceria.

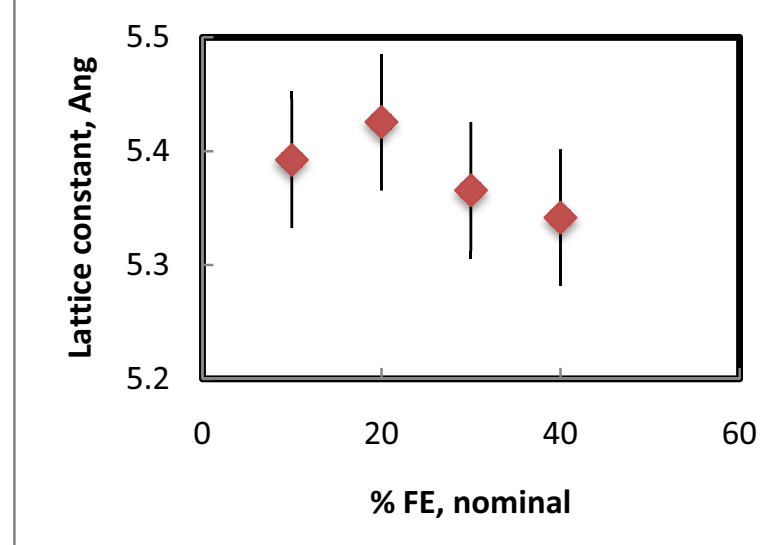

Fig. 3. Lattice constant vs Fe mole \%.
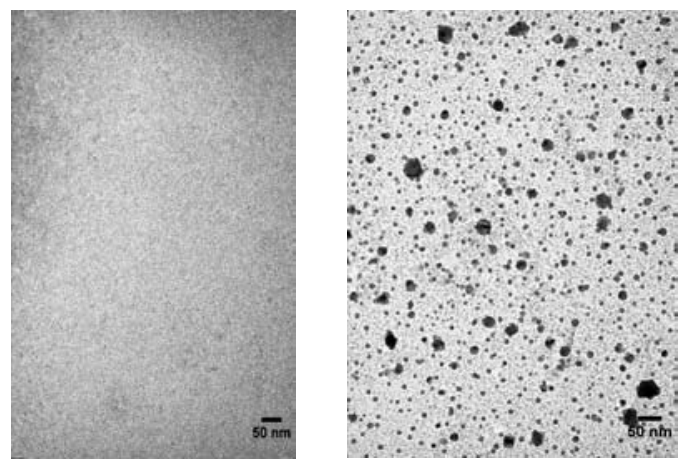

Fig. 5. Sample image before (left) and after (right) treatment for eight hours at $\sim 470{ }^{\circ} \mathrm{C}$.

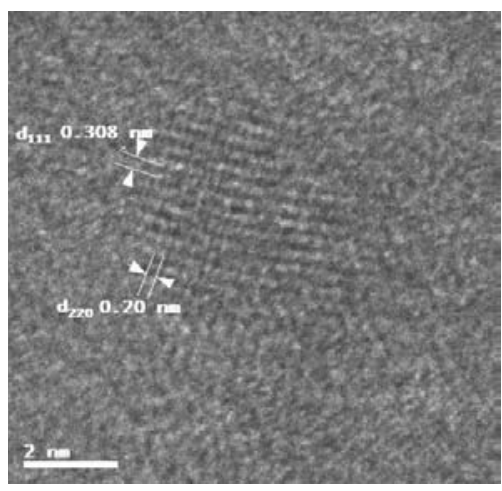

Fig. 2. HRTEM image of Fe-doped ceria with lattice plane spacings indicated.
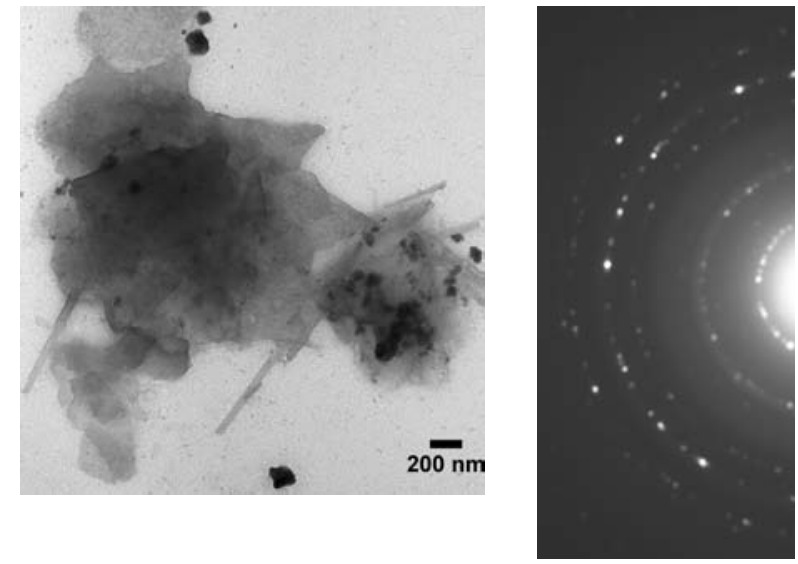

Fig. 4. Large crystalline particle with SAED consistent with 6 line ferrihydite.

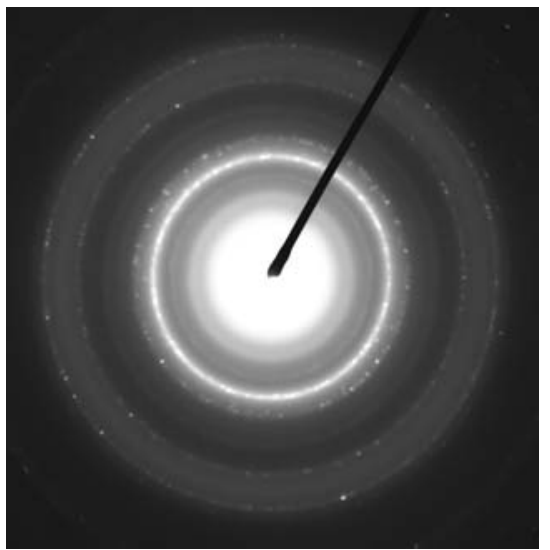

Fig. 6. SAED of Fe-doped ceria treated for eight hours at $\sim 470{ }^{\circ} \mathrm{C}$. Pattern can be indexed to a mixed phase of ceria, maghemite, and ferrihydrite. 